\title{
Honokiol attenuates lipotoxicity in hepatocytes via activating SIRT3-AMPK mediated lipophagy
}

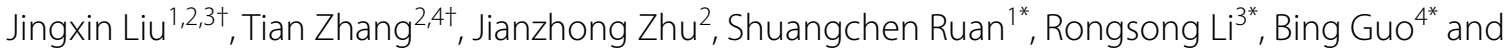
Ligen $\operatorname{Lin}^{2^{*}} \mathbb{D}$

\begin{abstract}
Background: Non-alcoholic fatty liver disease (NAFLD) is characterized by ectopic accumulation of triglycerides in the liver. Emerging evidence has demonstrated that lipophagy regulates lipid mobilization and energy homeostasis in the liver. Sirtuin 3 (SIRT3), a mitochondrial $\mathrm{NAD}^{+}$-dependent deacetylase, modulates the activities of several substrates involving in autophagy and energy metabolism. Honokiol (HK) is a natural lignan from the plants of Magnolia genus that exhibits potent liver protective property.

Methods: AML12 was challenged with $500 \mu \mathrm{M}$ palmitic acid and $250 \mu \mathrm{M}$ oleic acid mixture solution to induce lipotoxicity. C57BL/6J mice were fed with a choline-deficient high fat diet (CDHFD) to generate liver steatosis. The expression of autophagy-related and AMP-activated protein kinase (AMPK) pathway proteins was evaluated by Western blotting and immunofluorescence staining. Intracellular lipid accumulation was validated by Nile red staining. Molecular docking analysis was performed on AutoDock 4.2.

Results: HK (5 and $10 \mu \mathrm{M}$ ) was found to attenuate lipid accumulation through promoting SIRT3-AMPK-mediated autophagy, mainly on lipid droplets. HK had hydrophobic interaction with amino acid residues (PHE294, GLU323 and VAL324) and NAD ${ }^{+}$. Moreover, HK improved mitochondrial function to enhance lipolysis, through decreasing the acetylated long-chain acyl-CoA dehydrogenase level. In CDHFD-fed mice, HK (2.5 and $10 \mathrm{mg} / \mathrm{Kg}$ ) treatment obviously prevented lipid accumulation in the liver. And co-treatment of the AMPK inhibitor, Compound C, almost abolished the above changes.
\end{abstract}

\footnotetext{
*Correspondence: ruanshuangchen@sztu.edu.cn; lirongsong@sztu.edu.cn;

guobingbs@126.com; ligenl@um.edu.mo

${ }^{\dagger}$ Jingxin Liu and Tian Zhang contributed equally to this work

${ }^{1}$ College of Physics and Optoelectronic Engineering, Shenzhen

University, Shenzhen, Guangdong, China

${ }^{2}$ State Key Laboratory of Quality Research in Chinese Medicine,

Institute of Chinese Medical Sciences, University of Macau, Avenida da

Universidade, Taipa, Macao, SAR, China

${ }^{3}$ College of Health Science and Environmental Engineering, Shenzhen

Technology University, 3002 Lantian Road, Pingshan District, Shenzhen,

Guangdong, China

${ }^{4}$ The Department of Pathophysiology, Guizhou Provincial Key Laboratory

of Pathogenesis and Drug Research On Common Chronic Diseases),

College of Basic Medical Sciences, Guizhou Medical University,

Guiyang 550025, Guizhou, China
}

(c) The Author(s) 2021. Open Access This article is licensed under a Creative Commons Attribution 4.0 International License, which permits use, sharing, adaptation, distribution and reproduction in any medium or format, as long as you give appropriate credit to the original author(s) and the source, provide a link to the Creative Commons licence, and indicate if changes were made. The images or other third party material in this article are included in the article's Creative Commons licence, unless indicated otherwise in a credit line to the material. If material is not included in the article's Creative Commons licence and your intended use is not permitted by statutory regulation or exceeds the permitted use, you will need to obtain permission directly from the copyright holder. To view a copy of this licence, visit http://creativecommons.org/licenses/by/4.0/. The Creative Commons Public Domain Dedication waiver (http://creativeco mmons.org/publicdomain/zero/1.0/) applies to the data made available in this article, unless otherwise stated in a credit line to the data. 
Conclusions: These results suggest that HK could ameliorate lipotoxicity in hepatocytes by activating SIRT3-AMPKlipophagy axis, which might be a potential therapeutic agent against NAFLD.

Keywords: Honokiol, Hepatocytes, Lipid accumulation, Lipophagy, SIRT3, Lipolysis

\section{Background}

Non-alcoholic fatty liver disease (NAFLD) is prevailing in recent decades, which is closely related to non-alcoholic steatohepatitis (NASH), liver fibrosis and even hepatocellular carcinoma [1]. NAFLD is resulted from the ectopic accumulation of liver lipid, accompanied by lipotoxicity and subsequent metabolic abnormalities [2]. NAFLD occurs when the abnormal accumulation of triglycerides (TG) cannot compensate by the consumption [3]. Autophagy is a cellular recycling process that achieves energy homeostasis through lysosomal dependent degradation. The development of NAFLD is positively associated with impaired autophagy [4, 5]. Lipophagy describes such a process that lipid droplets (LDs) are engulfed into autolysosomes, causing the release of free fatty acids (FFAs) [6]. This actually opens up the possibility to alleviate lipotoxicity in hepatocytes.

Sirtuin 3 (SIRT3), mainly found in mitochondria, is a nicotinamide adenine dinucleotide $\left(\mathrm{NAD}^{+}\right)$-dependent deacetylase [7]. SIRT3 acts predominantly as a prosurvival factor to protect hepatocytes against oxidative stress [8]. We recently discovered that SIRT3-mediated autophagy promotes lipid mobilization in adipocytes via activating AMP-activated protein kinase (AMPK) [9]. Furthermore, SIRT3 activates lipophagy and chaperon-mediated autophagy to protect hepatocytes from lipotoxicity [10]. These findings imply that SIRT3 regulates lipid homeostasis and is a potential target for NAFLD. Unfortunately, the only ways to activate SIRT3 are calorie limitation and endurance training $[11,12]$. The small molecular SIRT3 activators are limited. Honokiol [2-(4-hydroxy-3-prop-2-enyl-phenyl)-4prop-2-enyl-phenol, HK], a natural lignan ubiquitous in the Magnolia genus, is traditional used in Asian ethnic medicines [13, 14]. HK was demonstrated to alleviate hepatic steatosis in various models [15-18]. HK acts as an activator of SIRT3 to reverse cardiac hypertrophy and alleviate oxidative stress [19-21]. Additionally, the binding of HK and SIRT3 activates AMPK to regulate in cellular energy homeostasis [20]. These findings suggest a possible role of $\mathrm{HK}$ in regulating hepatic lipid homeostasis.

To validate the hypothesis that HK stimulates lipid mobilization in hepatocytes by promoting SIRT3-mediated lipophagy, we evaluated the regulative effect of HK in lipid challenged hepatocytes and in the liver from choline-deficient high fat diet (CDHFD)-fed mice, and explored the role of SIRT3 in HK induced lipophagy and enhanced mitochondrial function.

\section{Materials and methods \\ Materials}

Dulbecco's Modified Eagle Medium (DMEM), penicillinstreptomycin $(\mathrm{P} / \mathrm{S})$, fetal bovine serum $(\mathrm{FBS})$, phosphatebuffered saline (PBS) and $0.25 \%(\mathrm{w} / \mathrm{v})$ trypsin-EDTA were purchased from Gibco (Gaithersburg, MD, USA). ITS-G $(5 \mathrm{mg} / \mathrm{mL}$ insulin, $5 \mathrm{mg} / \mathrm{L}$ transferrin, $5 \mu \mathrm{g} / \mathrm{L}$ selenious acid) was offered by Peiyuan Biotechnology (Shanghai, China). 3-(4,5-dimethylthiazol-2-yl)-2,5-diphenyltetrazolium bromide (MTT), HK, puromycin, oleic acid, palmitic acid, fatty acid free bovine serum albumin, isoproterenol, DAPI, Oil Red-O, and Free Glycerol Reagent were offered by Sigma-Aldrich (St. Louis, MO, USA). Lipofectamine 3000 Reagent, BCA protein assay kit, SuperSignal West Femto Maximum Sensitivity Substrate and Texas Red-conjugated secondary antibodies were bought from Thermo-Fisher (Grand Island, NY, USA). RIPA lysis buffer and ad-mCherry-GFPLC3 (\#C3011) were offered by Beyotime Biotechnology (Shanghai, China). Triton X-100 and PVDF membranes were supplied by Bio-Rad laboratories (Hercules, CA, USA). The shRNA targeting SIRT3 (mouse, sc-61556), scrambled shRNA (mouse, sc-1080600), and shRNA transfection reagent (mouse, sc-108061) were provided by Santa Cruz Biotechnology (Santa Cruz, CA, USA).

\section{Cell culture and treatments}

AML12 cells, obtained from ATCC (Rockville, MD, USA), were cultured in DMEM (supplemented with $10 \%$ FBS and ITS-G) in a humidified incubator $\left(5 \% \mathrm{CO}_{2}\right.$, $37{ }^{\circ} \mathrm{C}$ ). Palmitic acid and oleic acid were well dissolved with $75 \%(\mathrm{v} / \mathrm{v})$ ethanol at $55{ }^{\circ} \mathrm{C}$ and diluted to $500 \mu \mathrm{M}$ and $250 \mu \mathrm{M}$ with DMEM containing $1 \%$ fatty acid free bovine serum albumin $(\mathrm{w} / \mathrm{v})$, respectively. To make a mixture solution of palmtic acid and oleic acid $(\mathrm{P} / \mathrm{O})$, the two solutions were sterilized with $0.2 \mu \mathrm{m}$ filter membrane after shaking in an incubator for $2 \mathrm{~h}$.

\section{Cell viability}

The viability of AML12 cells was determined by MTT as previously described [10]. The working solution of HK was prepared immediately before use through diluting 
the stock solution (10 mM in DMSO) with fresh complete medium.

\section{Immunoblotting}

Protein concentration was quantified with a BCA Protein Assay Kit after lysing the cells with RIPA lysis buffer (containing 1\% protease inhibitor cocktail and 1\% phenylmethane sulfonylfflluoride). Equal amount of proteins $(20-30 \mu \mathrm{g})$ were separated using 5-12\% SDS-PAGE and then transferred to PVDF membranes. The membranes were firstly blocked with $5 \%$ defatted milk for $2 \mathrm{~h}$ at room temperature, followed by overnight incubation of specific primary antibodies at $4{ }^{\circ} \mathrm{C}$ and further incubation of secondary antibodies for $1 \mathrm{~h}$ at room temperature. SuperSignal West Femto Maximum Sensitivity Substrate kit was used to develop the signals. Visualization of the specific protein bands were achieved on the ChemiDoc MP Imaging System, and the bands were quantitated with Image Lab 5.1 (Bio-Rad laboratories, Hercules, CA, USA).

\section{RNA transfection and adenovirus infection}

Cells were transfected with $2 \mu \mathrm{g}$ shRNA using Lipofectamine 3000. After $6 \mathrm{~h}$, cells were switched into fresh medium and incubated for $24 \mathrm{~h}$. Then, cells were successively selected with puromycin $(2 \mu \mathrm{g} / \mathrm{mL})$ for 6 days and puromycin $(4 \mu \mathrm{g} / \mathrm{mL})$ for another 6 days. The survived cells were pooled together.

Cells $\left(2 \times 10^{5}\right)$ were seeded in 6-well plates and infected with $10 \mu \mathrm{L}$ Ad-mCherry-GFP-LC3 (multiplicity of infection $=5$ ) using Lipofectamine 3000. After $24 \mathrm{~h}$, the cells were switched to fresh medium a and incubated for an additional $24 \mathrm{~h}$. Then, cells were pooled together for further investigations.

\section{Confocal immunofluorescence microscopy}

Cells were fixed in formalin (10\%), blocked with goat serum (2.5\%), and incubated with primary antibodies at $4{ }^{\circ} \mathrm{C}$ overnight. Subsequently, cells were incubated with Texas Red-conjugated secondary antibody at room temperature for $2 \mathrm{~h}$. The nuclei were stained with DAPI. Leica TCS SP8 confocal fluorescence microscope (Leica, Buffalo Grove, IL, USA) was used to capture the images.

\section{Nile red staining}

Nile red staining was conducted as previously reported [22]. Briefly, AML12 hepatocytes were fixed with formaldehyde $(10 \%)$ and stained with Nile red $(1 \mu \mathrm{g} / \mathrm{mL})$. After incubating for $30 \mathrm{~min}$ at $4{ }^{\circ} \mathrm{C}$ and washing with PBS, the stained LDs were observed with fluorescence microscopy, and quantitated with flow cytometer with excitation and emission wavelength at 530 and $590 \mathrm{~nm}$, respectively.

\section{Determination of cellular triglycerides}

TG content in cell lysate and the liver tissue was determined by using commercial kits (Nanjing Jiancheng Bioengineering Institute, Nanjing, Jiangsu, China) and normalized by protein concentration.

\section{Molecular docking analysis}

Docking was performed on AutoDock 4.2. The crystal structure of the quaternary complex (SIRT3, a substrate, $\mathrm{NAD}^{+}$, and the specific agonist amiodarone hydrochloride; PDB ID: 5H4D) [23] was employed as the receptor. The protein was firstly prepared at $\mathrm{pH} 7.4$ with all the water molecules removed and corresponding hydrogen atoms added. The 3D structure of HK was downloaded from the PubChem database. Gasteiger charge was calculated and AD4 atom type was assigned, and a $50 \AA \times 48 \AA \times 40 \AA$ grid box with $375 \AA$ spacing was placed to include the surface of the catalytic cleft with the assistance of amiodarone hydrochloride. The genetic searching algorithm was chosen for docking calculations, and 50 genetic algorithm runs were performed. Other parameters were set as default. The acquired poses were clustered with a tolerance of $2.0 \AA$.

\section{Lipid droplets isolation}

LDs were isolated from AML12 hepatocytes as described previously [10]. Briefly, AML12 cells were lysed in hypotonic buffer $(50 \mathrm{mM}$ HEPES, $1 \mathrm{mM}$ EDTA and $2 \mathrm{mM}$ $\mathrm{MgCl}_{2}, \mathrm{pH}$ 7.4) supplemented with protease inhibitors after scaping and homogenized with 50 strokes in a Dounce homogenizer. After spinning down at 1,500 g for $5 \mathrm{~min}$, post-nuclear fractions were mixed with equal volume of $1.05 \mathrm{M}$ sucrose in isotonic buffer (50 mM HEPES, $100 \mathrm{mM} \mathrm{KCl}, 2 \mathrm{mM} \mathrm{MgCl} 2$ ) and centrifuged at 100,000 g for $2 \mathrm{~h}$ to remove Golgi, rough endoplasmic reticulum, mitochondria, and peroxisomes. The acquired supernatant was adjusted to $1 \mathrm{M}$ sucrose in hypotonic buffer and layered on a sucrose gradient $(1 \mathrm{~mL}$ of $0.75,0.5$, $0.25,0.125$, and $0 \mathrm{M}$ sucrose solution, respectively). The sucrose gradient tube was centrifuged at 100,000 $\mathrm{g}$ for $4 \mathrm{~h}$ at $4{ }^{\circ} \mathrm{C}$ afterwards, followed by collection of LD fractions from the top which were delipidated with acetone and washed with acetone/ether $(1: 1, \mathrm{v}: \mathrm{v})$. The pellet was dried under nitrogen and resuspended in protein lysis buffer. The protein concentration of LD fractions was analyzed by BCA Protein Assay kit, and subsequent western blotting was performed.

\section{Cellular thermal shift assay (CETSA)}

Cells were lysed after pretreatment with or without HK $(10 \mu \mathrm{M})$ for $12 \mathrm{~h}$. The lysates were centrifuged at $12,000 \mathrm{~g}$ for $10 \mathrm{~min}$ at $4{ }^{\circ} \mathrm{C}$ after incubating in ice for $10 \mathrm{~min}$. The 
protein concentration was determined and adjusted to $3 \mu \mathrm{g} / \mu \mathrm{L}$ using RIPA lysis buffer. Cell lysates $(50 \mu \mathrm{L})$ were transferred to new tubes and heated for $3 \mathrm{~min}$ at various temperature $\left(50-90{ }^{\circ} \mathrm{C}\right)$ on a thermal cycler. After standing in ice for $10 \mathrm{~min}$, soluble proteins were obtained by centrifugation at $12,000 \mathrm{~g}$ for $20 \mathrm{~min}$ at $4{ }^{\circ} \mathrm{C}$ and analyzed by western blotting [24].

\section{Mitochondrial membrane potential assay}

The fluorescent dye Rhodamine123 was employed to detect the mitochondrial membrane potential. Specifically, AML12 cells were cultured in the presence or absence of HK, and stained with Rhodamine123 $(10 \mu \mathrm{M})$ for $10 \mathrm{~min}$. Then, cells were washed twice with PBS, trypsinized and collected into a $1.5 \mathrm{~mL}$ tube. The change of membrane potential was qualitatively observed on an In Cell Analyzer 2000 (GE Healthcare Life Sciences, Chicago, IL, USA).

\section{Intracellular reactive oxygen species (ROS) detection}

Intracellular ROS levels were detected using DCFH-DA as previously described [25]. Briefly, cells $\left(1 \times 10^{5}\right)$ were seeded into 96-well black multitier plates (clear bottom) and then cultured overnight. The cells were treated with or without HK. After $12 \mathrm{~h}$, the cells were incubated with 2,7'-Dichlorodihydrofluorescein diacetate (DCFH-DA, Sigma-Aldrich, $10 \mu \mathrm{M})$ at $37^{\circ} \mathrm{C}$ in the dark for $15 \mathrm{~min}$. Fluorescence intensity was analyzed through FACS Calibur flow cytometry (BD, Lake Franklin, NJ, USA).

\section{Isoproterenol-stimulated lipolysis}

The lipolysis activity of AML12 cells was measured as described previously [26]. Cells were incubated with $10 \mu \mathrm{M}$ isoproterenol (stimulated condition) or DMSO (basal condition) at $37{ }^{\circ} \mathrm{C}$ for $2 \mathrm{~h}$. Subsequently, the medium was collected and heated at $85{ }^{\circ} \mathrm{C}$ for $10 \mathrm{~min}$. After centrifuged at 2,000 g for $10 \mathrm{~min}$, Clear supernatant $(10 \mu \mathrm{L})$ was used to determine the free glycerol content using Free Glycerol Reagent. Lipolysis activity was represented by glycerol concentrations and normalized by protein concentration.

\section{Immunoprecipitation}

Immunoprecipitation was performed as described previously [27]. Briefly, cell lysates (30 $\mu \mathrm{g}$ protein) were mixed with the indicated antibody $(2 \mu \mathrm{g})$ at $4{ }^{\circ} \mathrm{C}$ overnight. Then protein $\mathrm{A} / \mathrm{G}$-agarose beads $(20 \mu \mathrm{L})$ were added to the mixture and incubated on a rotator at $4{ }^{\circ} \mathrm{C}$ for $4 \mathrm{~h}$. Immune complexes were washed twice with lysis buffer supplemented with complete mini-protease inhibitor cocktail. Bound proteins were boiled in sample preparation buffer for $5 \mathrm{~min}$ and then immunoblotting was conducted.

\section{Ethic}

The procedures and operations involved in the animal experiments were conducted under the Animal Ethical and Welfare Committee of University of Macau (No. ICMS-AEC-2014-06) regulation. The male C57BL/6J mice were maintained in the animal facility of Faculty of Health Science, University of Macau. The mice were fed with normal chow diet (18\% protein, $4.5 \%$ fat, and $58 \%$ carbohydrate, Guangdong Medical Lab Animal Center, Guangzhou, Guangdong, China) and water ad libitum under standard conditions (specific-pathogen-free) with air filtration $\left(22 \pm 2{ }^{\circ} \mathrm{C}, 12\right.$-h light/12-h dark).

\section{Animal experimental procedure}

According to the body weight, twenty-eight male mice (6-8 weeks old) were randomly separated into 6 groups $(n=3-5)$. The vehicle group of mice (RD) were fed with a regular chow diet and intraperitoneally injected with $10 \mathrm{~mL} / \mathrm{kg}$ polyethylene glycol 400 (PEG 400, SigmaAldrich, St. Louis, MO, USA) solution (PEG 400:0.9\% saline, $6: 4, v / v)$. The remaining five groups of mice were fed with a choline-deficient high fat diet (Trophic Animal Feed High-Tech Co., Nantong, Jiangsu, China) and intraperitoneally injected with PEG 400 solution (CDHFD), $2.5 \mathrm{mg} / \mathrm{Kg}$ HK (HKL, $0.25 \mathrm{mg} / \mathrm{mL}$ HK in PEG 400 solution), $10 \mathrm{mg} / \mathrm{Kg}$ HK (HKH, $1 \mathrm{mg} / \mathrm{mL}$ HK in PEG 400 solution), $5 \mathrm{mg} / \mathrm{Kg}$ Compound C (CC, $0.5 \mathrm{mg} / \mathrm{mL}$ in PEG 400 solution), and the combination of $5 \mathrm{mg} / \mathrm{Kg} \mathrm{CC}$ and $10 \mathrm{mg} / \mathrm{Kg}$ HK $(\mathrm{CC}+\mathrm{HK}, 0.5 \mathrm{mg} / \mathrm{mL} \mathrm{CC}$ and $1 \mathrm{mg} / \mathrm{mL}$ HK in PEG 400 solution), respectively, once a day for consecutive 4 weeks. Blood samples were collected from tail vein under anesthesia $(0.5 \mathrm{~L} / \mathrm{min}$ inhalation of $3 \%$ isoflurane). The mice were euthanized by deeply inhaling carbon dioxide, and the livers were dissected.

\section{Determination of aspartate transaminase (AST) and alanine transaminase (ALT) levels}

The levels of AST and ALT in mouse serum were determined by using commercial assay kits (Nanjing Jiancheng, Nanjing, Jiangsu, China) in accordance with the manufacturer's protocols.

\section{H\&E staining and Oil-red $O$ staining of the liver}

After fixation in $4 \%$ paraformaldehyde, the liver was embedded in paraffin. $5 \mu \mathrm{m}$ sections were deparaffinized and rehydrated followed by hematoxylin and eosin (H\&E) staining and Oil-red $\mathrm{O}$ staining as described previously [28].

\section{Statistical analysis}

All experimental data were expressed as mean \pm S.D., and sample size for each experiment corresponds to three biological replicates. Data analysis was finished on 
GraphPad Prism-6 (GraphPad Software, San Diego, CA, USA), where significant differences between groups were evaluated by one-way analysis of variance (ANOVA) followed by Dunnet's multiple comparisons test $(p<0.05$ was considered as significant differences). Where statistical significance is evaluated, variance between groups is confirmed to be similar between comparison groups (control vs. experimental) and the statistical analysis is considered appropriate.

\section{Results}

\section{Honokiol attenuates lipid accumulation in lipotoxic hepatocytes through promoting autophagy}

$\mathrm{P} / \mathrm{O}$ (palmitic acid and oleic acid mixture) is widely used to induce lipotoxicity in vitro because of more efficient in inducing steatosis and lower cytotoxicity than palmitic acid alone $[29,30]$. Herein, lipid accumulation in AML12 cells was induced by stimulation with $\mathrm{P} / \mathrm{O}$ (the ratio of oleic acid to palmitic acid is 1:2). Firstly, we evaluated the effects of $\mathrm{HK}$ against $\mathrm{P} / \mathrm{O}$-induced lipid accumulation in AML12 cells. HK did not exhibit obvious cytotoxicity on AML12 cells up to $10 \mu \mathrm{M}$ [21]. Interestingly, a 2.7-fold increase of lipid content was observed after $\mathrm{P} / \mathrm{O}$ stimulation, and $\mathrm{HK}$ dose-dependently attenuated this effect, which was comparable with the positive control resveratrol $(10 \mu \mathrm{M})$, as indicated by Nile red staining and its quantitative analyses (Fig. 1A, B). P/O-induced increase of TG content was also reversed by HK treatment (Fig. 1C).

Impaired autophagy results in increased lipid storage in hepatocytes [31]. As shown in Fig. 1D, after P/O-treatment, there is a shrinkage in Beclin1 level and the ratio of LC3-II/LC3-I to approximately 48-75\%, whereas p62 level was elevated to $267 \%$, compared with those of the control cells, suggesting impaired autophagy in AML12 cells. Intriguingly, $\mathrm{HK}$ treatment reversed the above changes in dose-dependent manners, which was comparable with the positive control resveratrol (Fig. 1D). Meanwhile, HK enhanced autophagy in unstimulated AML12 cells, which was comparable with the positive control resveratrol (Fig. 1D). To check whether HK enhanced autophagic flux, the AML12 cells were infected with mRFP-GFP-LC3 adenovirus to label autophagosomal formation. As shown in Fig. $1 \mathrm{E}$ and $\mathrm{F}$, impaired autophagy was reflected by the decreased both red and green puncta in $\mathrm{P} / \mathrm{O}$ treated AML12 cells. More mRFP-LC3 puncta were observed in HK-treated cells as expected, suggesting that autophagic flux was improved with undisturbed lysosomal function and/or autophagosome-lysosome fusion. Furthermore, the fluorescent images indicated that HK-induced autophagosome formation was largely co-localized with LDs (Fig. 1G). These results indicated that $\mathrm{HK}$ mitigates lipid accumulation in lipotoxic hepatocytes through promoting autophagy.

\section{Honokiol attenuates lipid accumulation through SIRT3-mediated autophagy}

SIRT3 overexpression protects hepatocytes from lipotoxicity though promoting lipophagy and chaperonmediated autophagy [10]. Interestingly, $\mathrm{HK}$ treatment dose-dependently increased SIRT3 level in P/O-treated AML12 cells (Fig. 2A). To experimentally verify the interaction between HK and SIRT3 deacetylase, CETSA was performed on AML12 cells treated with or without HK. Compared to the control cells, the thermal stability of SIRT3 was strongly enhanced by HK at various temperatures (Fig. 2B). To verify the interaction pattern between HK and SIRT3, docking analysis was conducted. Clustering analysis showed the predominant cluster had the lowest binding energy with the best pose owing a $-7.2 \mathrm{kcal} /$ mol (Fig. 2C). HK had hydrophobic interaction with amino acid residues (PHE294, GLU323 and VAL324) and $\mathrm{NAD}^{+}$(Fig. 2D). Additionally, it was hydrogen bonded with an oxygen on the $\mathrm{NAD}^{+}$(Fig. 2D).

To evaluate the role of HK-driven SIRT3 in reducing lipid accumulation in hepatocytes, the SIRT3 knockdown AML12 cell line (SIRT3KD) was generated using shRNA targeting SIRT3. As expected, SIRT3 silencing partially blocked the lipid lowering effects of $\mathrm{HK}$ in $\mathrm{P} / \mathrm{O}$-stimulated AML12 cells (Fig. 2E). HK treatment increased the expression of LC3II, and reduced lipid content and LD size in P/O-treated AML12 cells; whereas, silencing of SIRT3 almost abrogated the effects of HK, which was comparable with the positive control resveratrol (Fig. 2F).

The LD fraction was isolated from scrambled and SIRT3KD AML12 cells treated with or without HK, and enrichment of LC3-II, Beclin1 and decreased p62 were observed in LDs, but not in homogenates after treatment

\footnotetext{
(See figure on next page.)

Fig. 1 HK attenuates lipid accumulation in lipotoxic hepatocytes through promoting autophagy. A Intracellular lipid content visualized with nile red (red) staining. Nuclei were stained with DAPI (blue). Scale bar $=10 \mu \mathrm{m}$. Resveratrol (10 $\mu \mathrm{M})$ was used as a positive control. B The lipid content determined by Nile red staining. C The cellular TG content. D Atg5, Beclin1, LC3 and p62 protein levels in P/O-stimulated AML12 hepatocytes treated without or with various concentrations of HK or $10 \mu \mathrm{M}$ resveratrol for $24 \mathrm{~h}$. GAPDH was used as a loading control. E AML12 cells were infected with the Ad-mCheryy-GFP-LC3, and treated with or without HK. The MRFP-LC3 and GFP-LC3 puncta were visualized on a confocal microscope. Scale bar $=10 \mu \mathrm{m}$. F The numbers of autophagosomes (yellow puncta) and autolysosomes (red puncta) were quantitated. G In P/O-stimulated AML12 hepatocytes, HK treatment induced more LC3-positive puncta (green) on LDs that were visualized with Nile red staining (red). Scale bar $=25 \mu \mathrm{m}$. Rapamycin was used as a positive control. Data represented means \pm SD. $n=6 .{ }^{*} p<0.05,{ }^{* *} p<0.01$ and ${ }^{* * *} p<0.001$, HK or resveratrol vs. P/O treatment. \#\#p<0.01, \#\#\#p<0.001, ctrl vs. P/O treatment. One-way ANOVA was used to calculate the $p$-values
} 

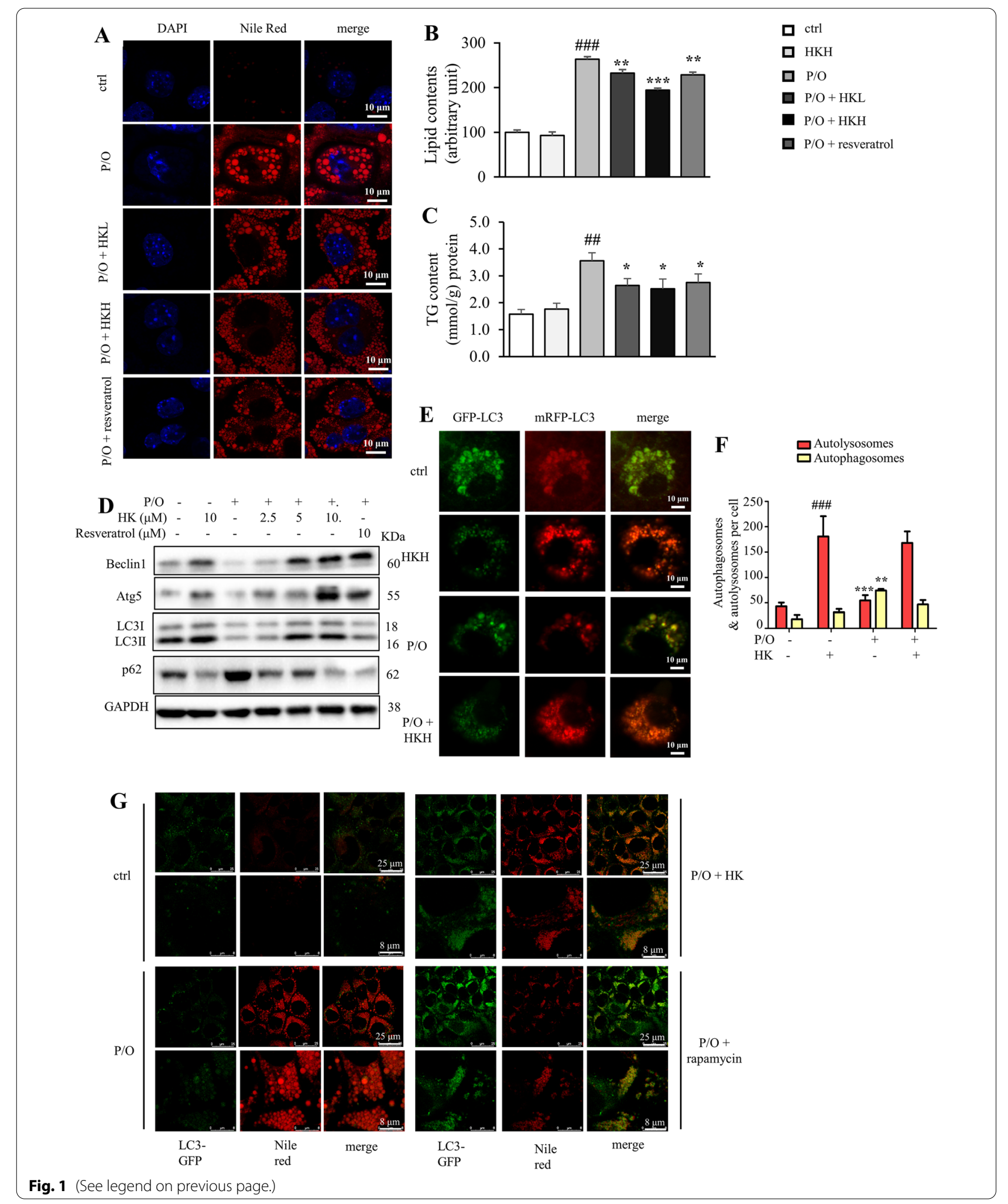

다 


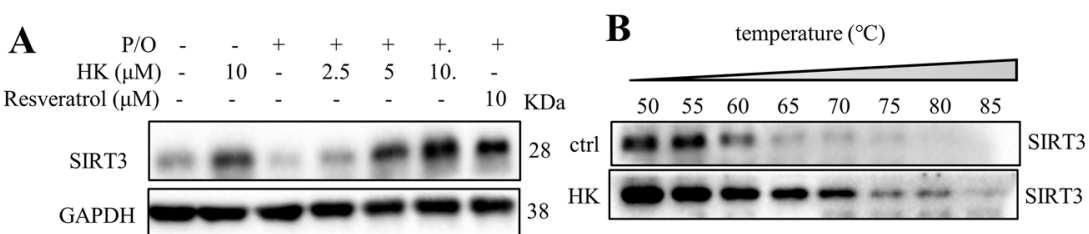

C

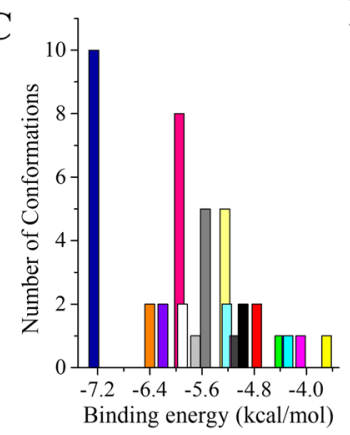

D

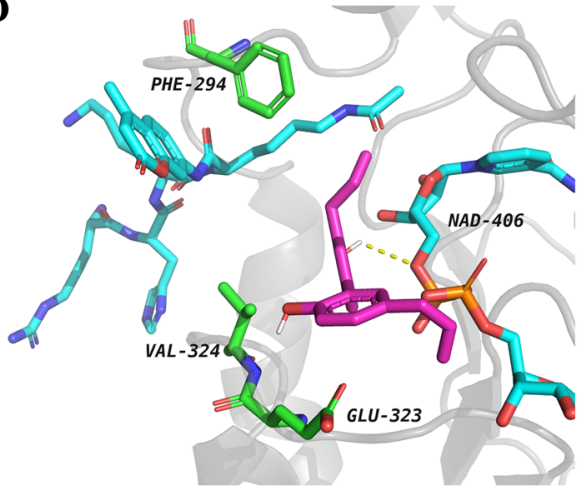

$\mathbf{F}$
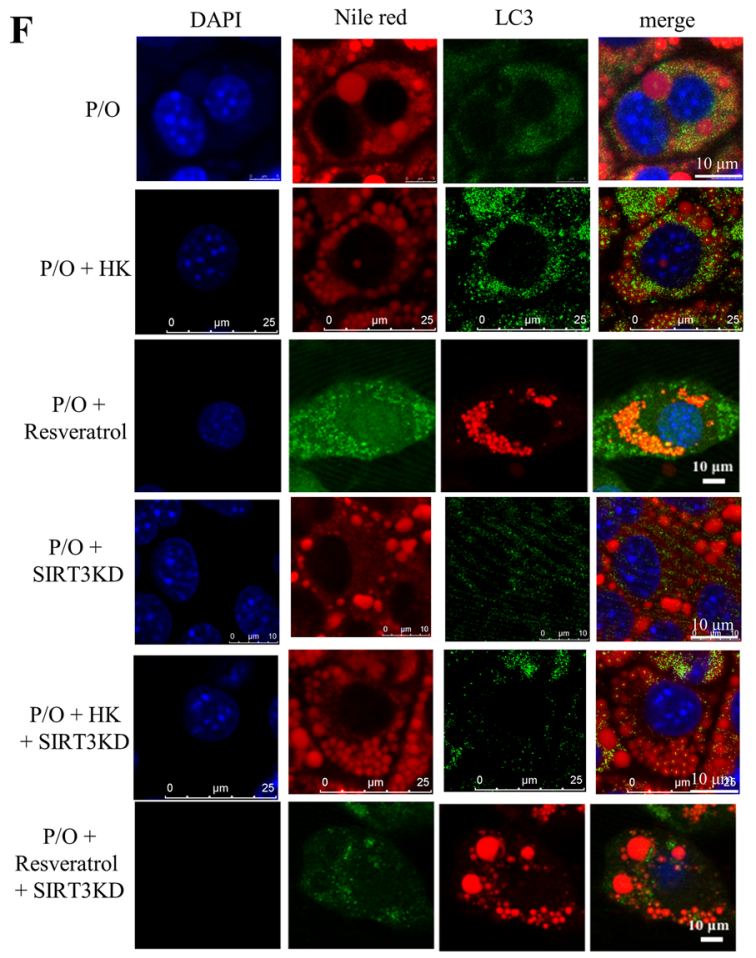

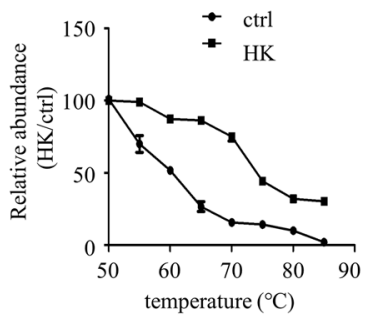

$\mathbf{E}$

口 scrambled

SIRT3KD
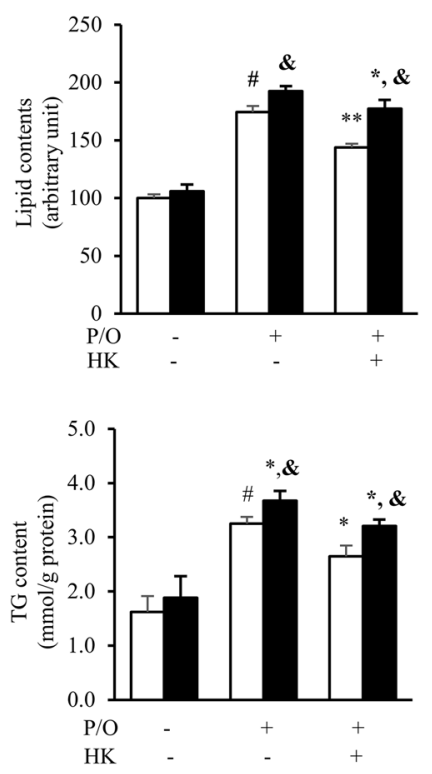

G

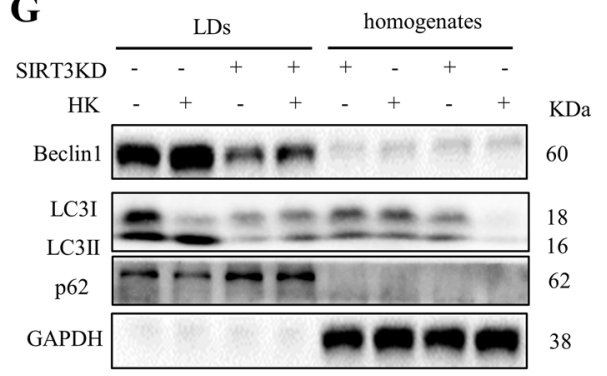

Fig. 2 HK attenuates lipid accumulation through SIRT3-mediated autophagy. P/O-stimulated AML12 cells were treated without or with various concentrations of HK for $24 \mathrm{~h}$. A SIRT3 protein levels were evaluated. GAPDH was used as a loading control. B CETSA was performed on AML12 cells treated with or without HK (10 $\mu \mathrm{M})$ for $12 \mathrm{~h}$. The SIRT3 protein levels were detected by using Western blotting. Data were normalized to the mean value of the respective group at $50^{\circ} \mathrm{C}(n=5)$. C Docking analysis of the binding between HK and SIRT3 (PDB ID: 5H4D). Cluster analysis of the docked conformations of HK. A tolerance of $2.0 \AA$ was used. D Interactions between HK and residues on SIRT3. The protein was shown in New Cartoon, and small molecules in sticks; the substrate (or NAD ${ }^{+}$), residues, and HK were colored in cyan, green, and magenta, respectively. E The scrambled and SIRT3KD cells were treated with or without HK for $24 \mathrm{~h}$. The lipid content was determined by flow cytometry after nile red staining and the cellular TG content were determined by commercial kit. F SIRT3 silencing blocked HK treatment-induced co-localization of LC3 puncta (green) on LDs. LDs were visualized with nile red fluorescence. Scale bar $=25 \mu \mathrm{m}$. G HK treatment activated autophagy mainly on LDs. Data was represented as means $\pm S D .{ }^{*} p<0.05$ and ${ }^{* *} p<0.01$, HK vs. P/O treatment. $\# p<0.05$, ctrl vs. P/O treatment. \& $p<0.05$, scrambled vs SIRT3KD groups. One-way ANOVA was used to calculate the $p$-values 
of HK; and deletion of SIRT3 almost reversed HK-driven activation of autophagy in the isolated LDs (Fig. 2G). These observations indicated HK treatment induced lipophagy rather than bulky autophagy to alleviate lipid accumulation and SIRT3 is required in HK-induced lipophagy.

\section{Honokiol alleviates lipid accumulation through SIRT3-AMPK-induced autophagy}

SIRT3 activates autophagy through the AMPK pathway in palmitate-stressed hepatocytes [10]. To elucidate the mechanism of HK on SIRT3-mediated autophagy, the phosphorylation level of AMPK was determined in HK treated hepatocytes. HK dose-dependently increased the phosphorylated AMPK level in P/O treated AML12 cells, which was comparable with the positive control resveratrol (Fig. 3A). Compound $\mathrm{C}(\mathrm{CC})$, an inhibitor to AMPK, remarkably diminished the effect of $\mathrm{HK}$ on activating autophagy (Fig. 3B). As shown in Fig. $3 \mathrm{C}$ and D, treatment of $\mathrm{CC}$ alone greatly increased lipid and TG contents, and co-treatment of $\mathrm{CC}$ and $\mathrm{HK}$ reversed the reducing effect of $\mathrm{HK}$ on lipid and TG contents, supporting that HK alleviated lipid accumulation in AML12 cells via activating AMPK signaling pathway. These results suggested that the effect of HK on lowering lipid accumulation was mediated through SIRT3-AMPK-mediated autophagy.

\section{Honokiol attenuates lipid accumulation by restoring mitochondrial function}

Next, we assumed that the lipid lowering effect of HK was involved in enhanced mitochondrial function. The mitochondrial membrane potential was evaluated by using Rhodamine 123 staining. The results showed that $\mathrm{P} / \mathrm{O}$ stimulation disrupted mitochondrial membrane potential, whereas HK-treated cells exhibited higher mitochondrial membrane potential, suggesting improved mitochondrial function (Fig. 4A). Meanwhile, lipid challenge led to high level of intracellular ROS and reduced mitochondrial content; whereas, HK treatment alleviated oxidative stress and slightly enhanced mitochondrial biogenesis in P/O-treated AML12 cells (Fig. 4B and

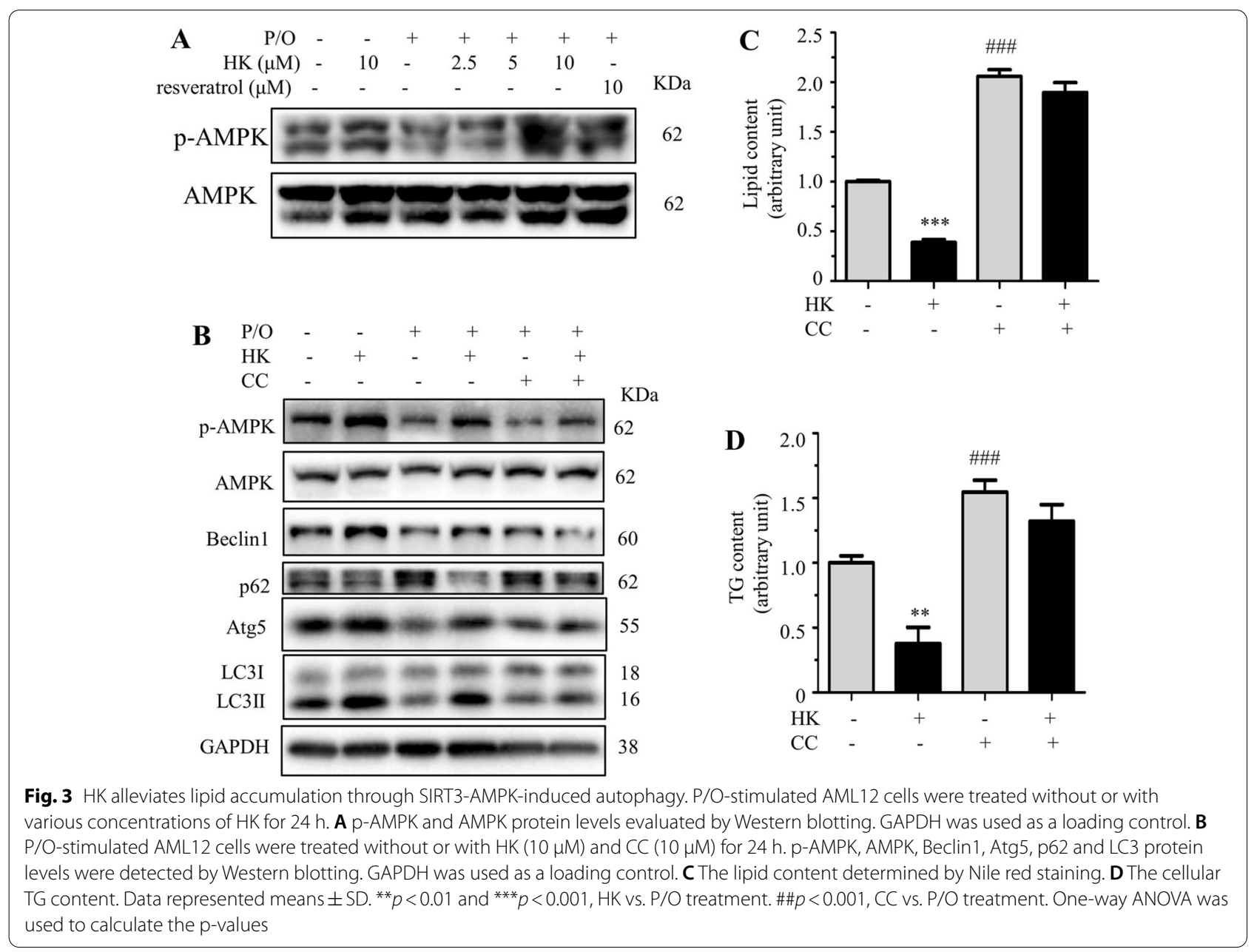



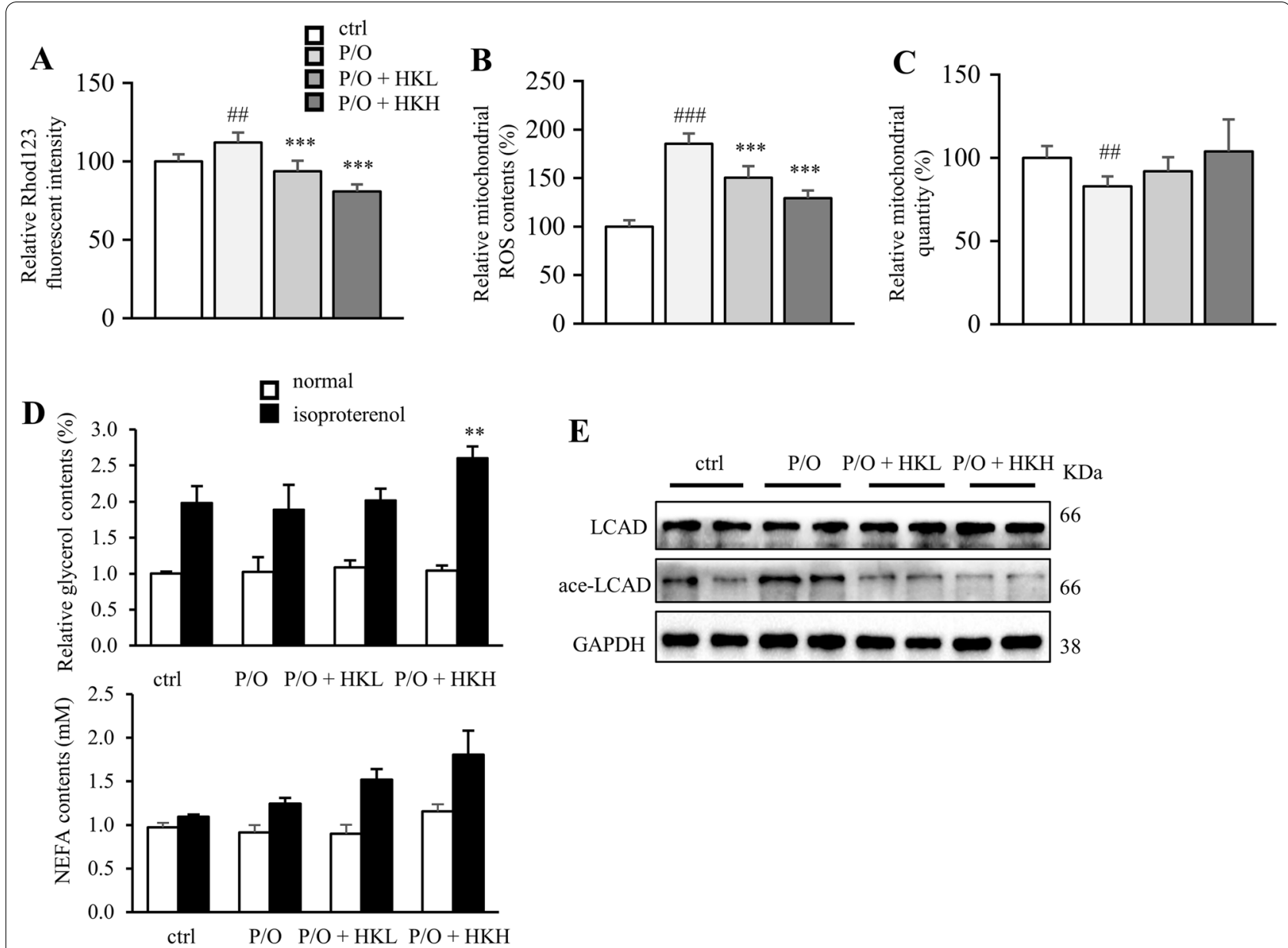

Fig. 4 HK attenuates lipid accumulation by restoring mitochondrial function. AML12 cells were treated with or without HK in the presence of P/O for $24 \mathrm{~h}$. A The relative Rhod 123 fluorescent intensity. B Intracellular ROS accumulation levels were determined by DCFH-DA assay. C Mitochondria were stained by Mitochondria-green and quantitated by flow cytometry. D Relative glycerol content in P/O-stimulated AML12 hepatocytes under both normal and isoproterenol-induced conditions. E The acetylated and total LCAD levels. Data was expressed as means \pm SD. ${ }^{* *} p<0.01$ and ${ }^{* * *} p<0.001$, HK vs. P/O treatment. \#\#p<0.01 and \#\#\#p<0.001 ctrl vs. P/O treatment. One-way ANOVA was used to calculate the $p$-values

C). Activation of autophagy not only shifts lipids to the lysosome for degradation by acid lipases, but enhances lipolysis by neutral lipases [10]. Upon isoproterenol stimulation, hormone-sensitive lipase translocates from the cytosol to the surfaces of intracellular LDs concomitant with the onset of lipolysis, as measured by the release of glycerol and non-esterified fatty acid (NEFA) to the culture medium. Interestingly, high dosage of HK enhanced the glycerol content in isoproterenol-stimulated cells, but not in the control cells (Fig. 4D); while HKH only slightly increased the NEFA level (Fig. 4D). Long-chain acyl-CoA dehydrogenase (LCAD) is a deacetylating substrate of SIRT3 [7]. HK decreased the acetylated LCAD level in a dose-dependently, but did not change the total LCAD level (Fig. 4E). Taken together, HK rescues hepatocytes from $\mathrm{P} / \mathrm{O}$-induced lipid accumulation by maintaining mitochondrial function and promoting lipolysis.

\section{HK alleviated liver steatosis in CDHFD-fed mice}

To investigate the protective effect of HK against lipotoxicity in vivo, CDHFD-induced liver steatosis mice were recruited (Fig. 5A). Four weeks of CDHFD feeding did not change body weight (data not shown). The serum levels of ALT and AST, as well as the liver TG content, were greatly increased in CDHFD-fed mice when compared with those of RD-fed mice (Fig. 5B-D), which suggested the liver steatosis model was well generated. Furthermore, the H\&E staining and Oil-red $O$ staining of the liver from CDHFD-fed mice exhibited a great increase of lipid content (Fig. 5E and F). As expected, either low or high dosage of HK treatment markedly decreased the serum ALT and AST levels, and the liver TG and lipid contents, compared 


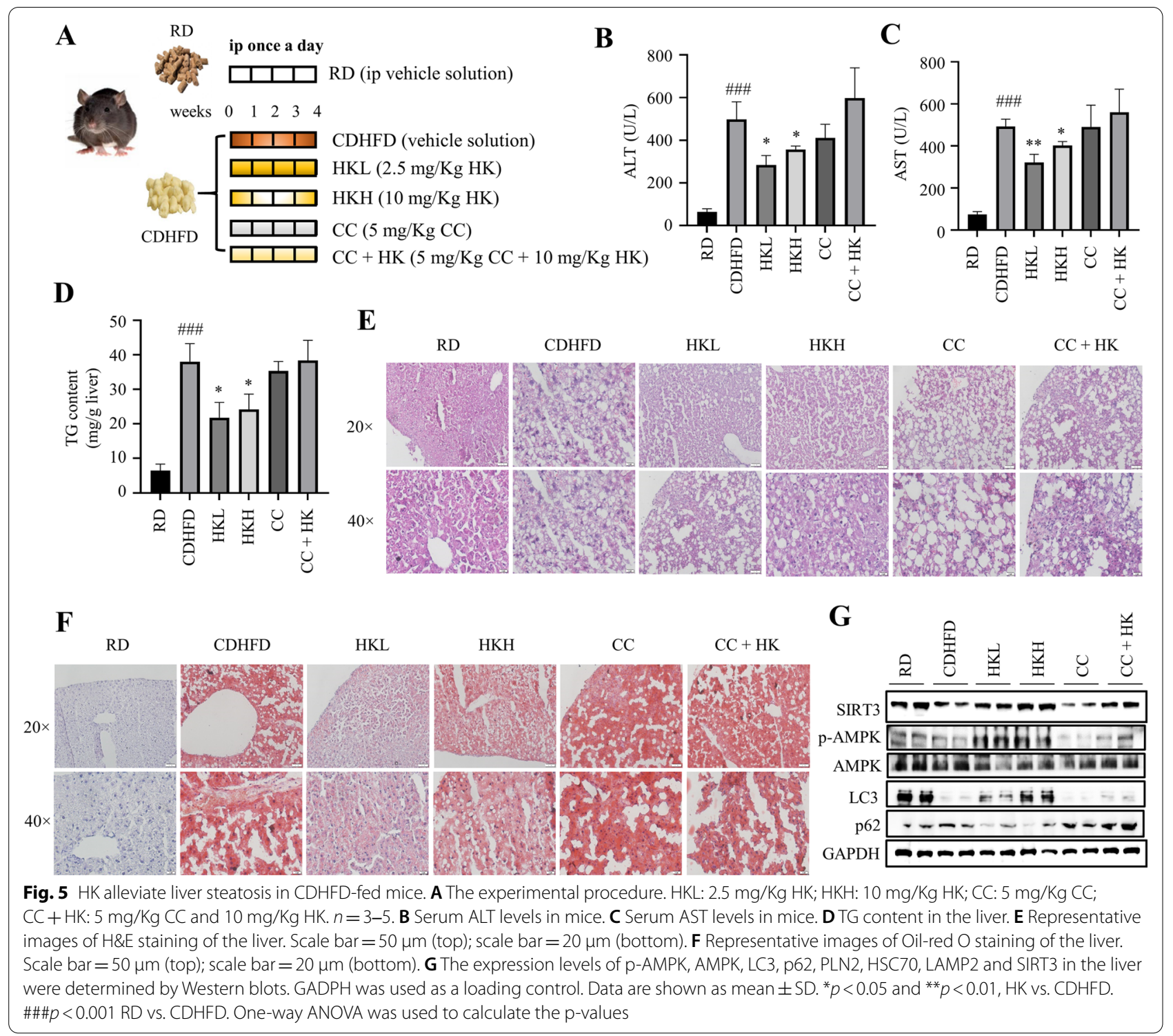

with those of CDHFD mice (Fig. 5B-F). These changes were abolished in CC co-treated group (Fig. 5B-F). The phosphorylation of AMPK and the protein level of SIRT3 were suppressed in the liver from CDHFD mice (Fig. 5G), and HKL and HKH activated AMPK and increased the protein expression of SIRT3 in the liver (Fig. 5G). On the other hand, CDHFD feeding resulted decreased protein expression of LC3 and increased protein expression of p62 in the liver, suggesting impaired autophagy (Fig. 5G). Intriguingly, either low or high dosage of HK treatment activated autophagy in the liver (Fig. 5G). And co-treatment of CC almost reversed the above changes (Fig. 5G). Collectively, these results suggested that HK alleviates liver steatosis in CDHFD-fed mice, which might be mediated through the SIRT3AMPK axis.

\section{Discussion}

Increasing evidence has uncovered a positive connection between lipophagy and the onset of NAFLD. Organisms regulate free fatty acids release to supply metabolic demand by lipophagy [32]. Impaired autophagy leads to excessive lipid accumulation in the liver to cause hepatic steatosis [33, 34]. HK has been found to activate autophagy in several cancer cells [35-37]. Herein, we found $\mathrm{HK}$ attenuates lipid accumulation in lipotoxic hepatocytes through promoting SIRT3-AMPK-induced autophagy and mitochondrial function. Furthermore, the 
role of HK in treating NAFLD was verified in a CDHFDinduced fatty liver mice model.

Autophagy accounts for a major part of lipolysis in liver. Consequently, autophagy blockage through knockdown of the key autophagic genes like Atgs in hepatocytes, led to an increase of LDs in cells even under normal nutritional conditions [38]. Interestingly, impaired autophagy further deteriorated LDs accumulation, leading to hepatotoxicity and severe steatosis [39]. In our current study, HK was found to activate lipophagy, stimulate lipolysis under isoproterenol treated condition and decrease the acetylated LCAD level. These results indicated that HK protects hepatocytes against lipotoxicity through enhancing lipophagy and lipolysis.

Liver contains a large number of mitochondria, which are the predominant source of intracellular ROS. Excessive ROS accumulation results in cell death through the oxidation of polyunsaturated fatty acids [40]. Mitochondrial homeostasis was interrupted when the hepatocytes were exposed to $\mathrm{P} / \mathrm{O}$ stimulation, accompanied with decreased mitochondrial content and increased ROS production. Interestingly, high dose of HK maintained mitochondrial integrity in hepatocytes under lipotoxic stress. In fact, mitochondrial biogenesis acts as a critical factor for mitochondrial quantity, and HK was found to facilitate the process by targeting peroxisome proliferator-activated receptor- $\gamma$ coactivator- $1 \alpha$ [41]. Herein, HK did not affect mitochondrial biogenesis, but enhanced mitochondrial function in lipotoxic hepatocytes. SIRT3 may also be involved in mitochondrial renewal and hepatocyte proliferation through mitophagy mechanisms [42]. Future studies are required to fully elucidate how HK regulates mitophagy in the context of hepatocellular lipotoxicity.

In response to a variety of conditions that deplete cellular energy levels, AMPK is activated to coordinate metabolic pathways and balance nutrient supply with energy demand [43]. Previous studies indicated that AMPK activation attenuates hepatic steatosis through suppressing de novo lipogenesis in hepatocytes, increasing fatty acid oxidation in the liver, and promoting mitochondrial function and integrity in adipose tissue [44]. Ablation of AMPK activity in adipose tissue causes a decrease in adipose tissue insulin sensitivity and an increase in liver lipid accumulation [45]. CC is widely used as a cell-permeable ATP-competitive inhibitor of AMPK to revert the positive effects of 5 -aminoimidazole-4-carboxamide-1- $\beta$ D-ribofuranoside and metformin [46]. CC treatment was reported to increase lipid and TG contents in hepatocytes [47], which is consistent with the current observation. On the contrary, CC has been demonstrated to be with AMPK-independent pharmacological actions. In HFD-fed mice, CC administration was found to reduce

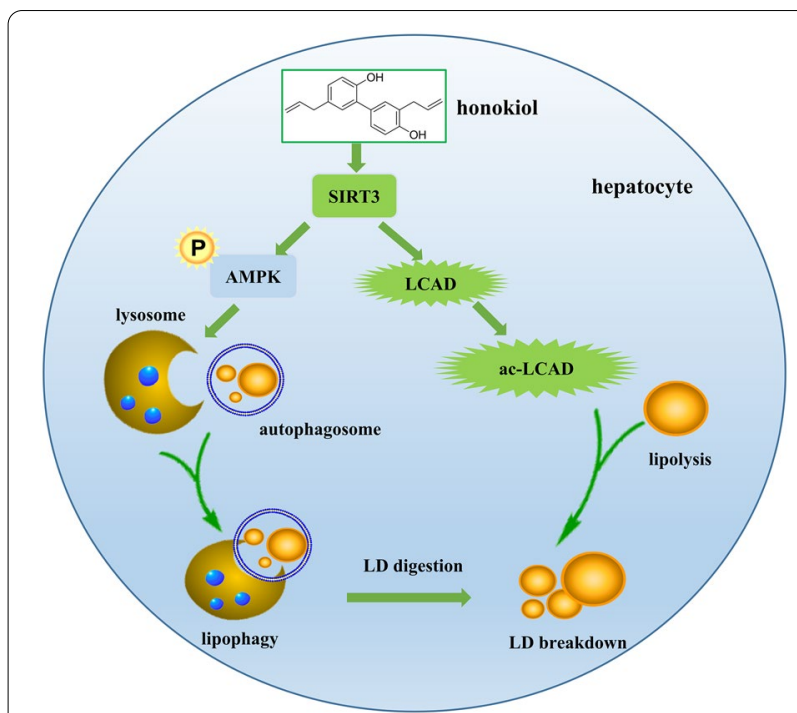

Fig. 6 Schematic of the role of HK in protecting hepatocytes against lipotoxicity. HK activates SIRT3, which in turn activates AMPK to enhance autophagy on LDs and deacetylates LCAD to increase fatty acid oxidation in mitochondria, resulting in attenuation of lipotoxicity in hepatocytes

hepatic steatosis and ballooning by impairing NOD-, LRR- and pyrin domain-containing protein 3 inflammasome activation and the related inflammation [48].

The most widespread and prevailing model describing the development of NAFLD is the "multiple-hit hypothesis", where the "first hit", hepatic lipid accumulation, induces lipotoxicity or steatosis, rendering liver more vulnerable to "subsequent hits" injury, such as mitochondrial dysfunction and oxidative stress, which in turn leads to steatohepatitis and cirrhosis, and eventually hepatocellular carcinoma [49]. In the previous study, we reported that HK scavenges excessive ROS and repairs cellular damages in oxidative injured hepatocytes. Herein, we further found that HK activates lipophagy to promote LD breakdown, leading to reduced lipotoxicity in hepatocytes. Taken together, HK might protect hepatocytes against oxidative stress and alleviate lipotoxicity, rendering it a potential candidate to treat NAFLD against multiple hits.

\section{Conclusions}

In conclusion, we verified HK protects hepatocytes against lipotoxic stress through enhancing SIRT3AMPK-induced lipophagy, and maintain mitochondrial morphology and integrity (Fig. 6). HK could be a potential candidate in the treatment of NAFLD.

\section{Abbreviations}

ALT: Aminoalanine transaminase; AMPK: AMP-activated protein kinase; AST: Aspartate transaminase; CC: Compound C; CDHFD: Choline-deficient high fat 
diet; CETSA: Cellular thermal shift assay; FFAs: Free fatty acids; HCC: HepatocelIular carcinoma; HK: Honokiol; LCAD: Long-chain acyl-CoA dehydrogenase; LD; Lipid droplet; 3-MA: 3-Methyladenine; NASH: Non-alcoholic steatohepatitis; NAFLD: Non-alcoholic fatty liver disease; P/O: Palmitic acid/oleic acid; ROS: Reactive oxygen species; SIRT3: Sirtuin 3; TG: Triglyceride.

\section{Acknowledgements}

The authors thank the technical team of Institute of Chinse Medical Sciences in University of Macau for their valuable assistance.

\section{Authors' contributions}

JL: Conceptualization, Investigation, Review \& editing. TZ: Conceptualization, Investigation, Writing original draft. JZ: Investigation, Review \& editing. SR, RL, BG and LL: Conceptualization, Methodology, Validation, Writing review \& editing, Supervision.

\section{Funding}

Financial support by the Science and Technology Development Fund, Macao SAR (File no. FDCT 0031/2019/A1 to LL), the Research Fund of University of Macau (MYRG2017-00109-ICMS and MYRG2018-00037-ICMS to LL), National Natural Science Foundation of China (82100623 to TZ and 81872754 to LL), the Special anti-COVID-19 Project Fund of Guangdong Provincial Education Department (2020KZDZX1214 to RL), Natural Science Foundation of Top Talent of SZTU (2020102 to RL), the Shenzhen Natural Science Foundation (JCYJ20190813141001745 to RL), and the Startup Research Fund of Shenzhen Technology University (RL) are gratefully acknowledged.

\section{Availability of data and materials}

Not applicable.

\section{Declarations}

\section{Ethics approval and consent to participate}

Not applicable.

\section{Consent for publication}

Not applicable.

\section{Competing interests}

The authors declare that they have no competing interests.

Received: 10 August 2021 Accepted: 31 October 2021

Published online: 10 November 2021

\section{References}

1. Lu FB, Zheng Kl, Rios RS, Targher G, Byrne CD, Zheng MH. Global epidemiology of lean non-alcoholic fatty liver disease: a systematic review and meta-analysis. J Gastroenterol Hepatol. 2020;35(12):2041-50.

2. Cohen JC, Horton JD, Hobbs HH. Human fatty liver disease: old questions and new insights. Science. 2011;332(6037):1519-23.

3. Liang Z, Chen X, Shi J, Hu H, Xue Y, Ung COL. Efficacy and safety of traditional Chinese medicines for non-alcoholic fatty liver disease: a systematic literature review of randomized controlled trials. Chin Med. 2021;16(1):9.

4. Gonzalez-Rodriguez A, Mayoral R, Agra N, Valdecantos MP, Pardo V, Miquilena-Colina ME, et al. Impaired autophagic flux is associated with increased endoplasmic reticulum stress during the development of NAFLD. Cell Death Dis. 2014;5:e1179.

5. Baerga R, Zhang Y, Chen PH, Goldman S, Jin S. Targeted deletion of autophagy-related 5 (atg5) impairs adipogenesis in a cellular model and in mice. Autophagy. 2009;5(8):1118-30.

6. Singh R, Kaushik S, Wang Y, Xiang Y, Novak I, Komatsu M, et al. Autophagy regulates lipid metabolism. Nature. 2009;458(7242):1131-5.

7. Hirschey MD, Shimazu T, Goetzman E, Jing E, Schwer B, Lombard DB, et al. SIRT3 regulates mitochondrial fatty-acid oxidation by reversible enzyme deacetylation. Nature. 2010;464(7285):121-5

8. Ma C, Sun Y, Pi C, Wang H, Sun H, Yu X, et al. Sirt3 attenuates oxidative stress damage and rescues cellular senescence in rat bone marrow mesenchymal stem cells by targeting superoxide dismutase 2. Front Cell Dev Biol. 2020;8:599376.

9. Zhang T, Liu J, Tong Q, Lin L. SIRT3 acts as a positive autophagy regulator to promote lipid mobilization in adipocytes via activating AMPK. Int J Mol Sci. 2020;21(2):372.

10. Zhang T, Liu J, Shen S, Tong Q, Ma X, Lin L. SIRT3 promotes lipophagy and chaperon-mediated autophagy to protect hepatocytes against lipotoxicity. Cell Death Differ. 2020;27(1):329-44.

11. Qiu X, Brown K, Hirschey MD, Verdin E, Chen D. Calorie restriction reduces oxidative stress by SIRT3-mediated SOD2 activation. Cell Metab. 2010;12(6):662-7.

12. Vassilopoulos A, Pennington JD, Andresson T, Rees DM, Bosley AD, Fearnley IM, et al. SIRT3 deacetylates ATP synthase F1 complex proteins in response to nutrient- and exercise-induced stress. Antioxid Redox Signal. 2014;21(4):551-64.

13. Chou PY, Chang WC, Liu FC, Lan SJ, Sheu MJ, Chen JS. Honokiol, an active compound of Magnolia officinalis, is involved in restoring normal baroreflex sensitivity in hypercholesterolemic rabbits. Food Sci Nutr. 2020;8(2):1093-103.

14. Chen C, Zhang QW, Ye Y, Lin LG. Honokiol: A naturally occurring lignan with pleiotropic bioactivities. Chin J Nat Med. 2021;19(7):481-90.

15. Yin HQ, Kim YC, Chung YS, Kim YC, Shin YK, Lee BH. Honokiol reverses alcoholic fatty liver by inhibiting the maturation of sterol regulatory element binding protein-1c and the expression of its downstream lipogenesis genes. Toxicol Appl Pharmacol. 2009;236(1):124-30.

16. Jeong YH, Hur HJ, Jeon EJ, Park SJ, Hwang JT, Lee AS, et al. Honokiol improves liver steatosis in ovariectomized mice. Molecules. 2018;23(1):194.

17. Zhai T, Xu W, Liu Y, Qian K, Xiong Y, Chen Y. Honokiol alleviates methionine-choline deficient diet-induced hepatic steatosis and oxidative stress in C57BL/6 mice by regulating CFLAR-JNK pathway. Oxid Med Cell Longev. 2020;2020:2313641.

18. Seo MS, Kim JH, Kim HJ, Chang KC, Park SW. Honokiol activates the LKB1-AMPK signaling pathway and attenuates the lipid accumulation in hepatocytes. Toxicol Appl Pharmacol. 2015;284(2):113-24.

19. Pillai VB, Samant S, Sundaresan NR, Raghuraman H, Kim G, Bonner MY, et al. Honokiol blocks and reverses cardiac hypertrophy in mice by activating mitochondrial Sirt3. Nat Commun. 2015;6:6656.

20. Caballero EP, Mariz-Ponte N, Rigazio CS, Santamaria MH, Corral RS. Honokiol attenuates oxidative stress-dependent heart dysfunction in chronic Chagas disease by targeting AMPK / NFE2L2 / SIRT3 signaling pathway. Free Radic Biol Med. 2020;156:113-24.

21. Liu JX, Shen SN, Tong Q, Wang YT, Lin LG. Honokiol protects hepatocytes from oxidative injury through mitochondrial deacetylase SIRT3. Eur J Pharmacol. 2018;834:176-87.

22. Shen S, Liao Q, Zhang T, Pan R, Lin L. Myricanol modulates skeletal muscle-adipose tissue crosstalk to alleviate high-fat diet-induced obesity and insulin resistance. Br J Pharmacol. 2019;176(20):3983-4001.

23. Zhang J, Zou L, Shi D, Liu J, Zhang J, Zhao R, et al. Structureguided design of a small-molecule activator of Sirtuin-3 that modulates autophagy in triple negative breast cancer. J Med Chem. 2021;64(19):14192-216.

24. Jafari $R$, Almqvist $H$, Axelsson $H$, lgnatushchenko $M$, Lundback T, Nordlund $P$, et al. The cellular thermal shift assay for evaluating drug target interactions in cells. Nat Protoc. 2014;9(9):2100-22.

25. Liu JX, Li D, Zhang T, Tong Q, Ye RD, Lin LG. SIRT3 protects hepatocytes from oxidative injury by enhancing ROS scavenging and mitochondrial integrity. Cell Death Dis. 2017;8:e3158.

26. Lin LG, Lee JH, Bongmba OYN, Ma XJ, Zhu XW, Sheikh-Hamad D, et al. The suppression of ghrelin signaling mitigates age-associated thermogenic impairment. Aging. 2014;6(12):1019-32.

27. Li D, Liu QY, Sun W, Chen XP, Wang Y, Sun YX, et al. 1,3,6,7-Tetrahydroxy8-prenylxanthone ameliorates inflammatory responses resulting from the paracrine interaction of adipocytes and macrophages. Br J Pharmacol. 2018;175(10):1590-606.

28. Gao S, Wang TZ, Huang XK, Jin YQ, XU Y, Xi YM, Wang JW, et al. Exploring the protective effect of Modified Xiaochaihu Decoction against hepatic steatosis and inflammation by network pharmacology and validation in ageing rats. Chin Med. 2020;15(1):96.

29. Ricchi M, Odoardi MR, Carulli L, Anzivino C, Ballestri S, Pinetti A, et al. Differential effect of oleic and palmitic acid on lipid accumulation 
and apoptosis in cultured hepatocytes. J Gastroenterol Hepatol. 2009;24(5):830-40.

30. Moravcova A, Cervinkova Z, Kucera O, Mezera V, Rychtrmoc D, Lotkova H. The effect of oleic and palmitic acid on induction of steatosis and cytotoxicity on rat hepatocytes in primary culture. Physiol Res. 2015;64(Suppl 5):S627-36

31. Zhang Y, Goldman S, Baerga R, Zhao Y, Komatsu M, Jin S. Adipose-specific deletion of autophagy-related gene 7 (atg7) in mice reveals a role in adipogenesis. Proc Natl Acad Sci USA. 2009;106(47):19860-5.

32. Rambold AS, Cohen S, Lippincott-Schwartz J. Fatty acid trafficking in starved cells: regulation by lipid droplet lipolysis, autophagy, and mitochondrial fusion dynamics. Dev Cell. 2015;32(6):678-92.

33. Zhang LQ, Zhang ZG, Li CB, Zhu TT, Gao J, Zhou H, Liang B, et al. S100A11 promotes liver steatosis via FOXO1-mediated autophagy and lipogenesis. Cell Mol Gastroenterol Hepatol. 2021;11(3):697-724.

34. Dai XM, Feng JY, Chen Y, Huang S, Shi XF, Liu X, Sun Y. Traditional Chinese Medicine in nonalcoholic fatty liver disease: molecular insights and therapeutic perspectives. Chin Med. 2021;16(1):68.

35. Lin MC, Lee YW, Tseng YY, Lin YW, Chen JT, Liu SH, et al. Honokiol induces autophagic apoptosis in neuroblastoma cells through a P53-dependent pathway. Am J Chin Med. 2019;47(4):895-912.

36. Li Z, Dong H, Li M, Wu Y, Liu Y, Zhao Y, et al. Honokiol induces autophagy and apoptosis of osteosarcoma through PI3K/Akt/mTOR signaling pathway. Mol Med Rep. 2018;17(2):2719-23.

37. Huang $K$, Chen Y, Zhang R, Wu Y, Ma Y, Fang X, et al. Honokiol induces apoptosis and autophagy via the ROS/ERK1/2 signaling pathway in human osteosarcoma cells in vitro and in vivo. Cell Death Dis. 2018;9(2):157.

38. Zechner R, Madeo F, Kratky D. Cytosolic lipolysis and lipophagy: two sides of the same coin. Nat Rev Mol Cell Biol. 2017;18(11):671-84.

39. Schulze RJ, Sathyanarayan A, Mashek DG. Breaking fat: The regulation and mechanisms of lipophagy. Biochim Biophys Acta Mol Cell Biol Lipids. 2017;1862(10 Pt B):1178-87.

40. Jaeschke H, Ramachandran A. Reactive oxygen species in the normal and acutely injured liver. J Hepatol. 2011;55(1):227-8.
41. Brenmoehl J, Hoeflich A. Dual control of mitochondrial biogenesis by sirtuin 1 and sirtuin 3. Mitochondrion. 2013;13(6):755-61.

42. Li R, Xin T, Li D, Wang C, Zhu H, Zhou H. Therapeutic effect of Sirtuin 3 on ameliorating nonalcoholic fatty liver disease: the role of the ERK-CREB pathway and Bnip3-mediated mitophagy. Redox Biol. 2018;18:229-43.

43. Kahn BB, Alquier T, Carling D, Hardie DG. AMP-activated protein kinase: ancient energy gauge provides clues to modern understanding of metabolism. Cell Metab. 2005;1 (1):15-25.

44. Li Y, Xu S, Mihaylova MM, Zheng B, Hou X, Jiang B, Zang M, et al. AMPK phosphorylates and inhibits SREBP activity to attenuate hepatic steatosis and atherosclerosis in diet-induced insulin-resistant mice. Cell Metab. 2011;13(4):376-88

45. Mottillo EP, Desjardins EM, Crane JD, Smith BK, Green AE, Ducommun S, Steinberg GR, et al. Lack of adipocyte AMPK exacerbates insulin resistance and hepatic steatosis through brown and beige adipose tissue function. Cell Metab. 2016;24(1):118-29.

46. Latorre J, Ortega FJ, Liñares-Pose L, Moreno-Navarrete JM, Lluch A, Comas F, Fernández-Real JM. Compounds that modulate AMPK activity and hepatic steatosis impact the biosynthesis of microRNAs required to maintain lipid homeostasis in hepatocytes. EBioMedicine. 2020;53:102697.

47. Xu N, Luo H, Li M, Wu J, Wu X, Chen L, Liu Y, et al. beta-patchoulene improves lipid metabolism to alleviate non-alcoholic fatty liver disease via activating AMPK signaling pathway. Biomed Pharmacother. 2021;134:111104

48. Wang F, Liu YX, Yuan JJ, Yang WJ, Mo ZH. Compound C protects mice from HFD-induced obesity and nonalcoholic fatty liver disease. Int J Endocrinol. 2019;2019:3206587.

49. Buzzetti E, Pinzani M, Tsochatzis EA. The multiple-hit pathogenesis of nonalcoholic fatty liver disease (NAFLD). Metabolism. 2016;65(8):1038-48.

\section{Publisher's Note}

Springer Nature remains neutral with regard to jurisdictional claims in published maps and institutional affiliations.
Ready to submit your research? Choose BMC and benefit from:

- fast, convenient online submission

- thorough peer review by experienced researchers in your field

- rapid publication on acceptance

- support for research data, including large and complex data types

- gold Open Access which fosters wider collaboration and increased citations

- maximum visibility for your research: over 100M website views per year

At $\mathrm{BMC}$, research is always in progress.

Learn more biomedcentral.com/submissions 